Urol Int

DOI: $10.1159 / 000346748$
Received: September 21, 2012

Accepted after revision: December 20, 2012 Published online: $\mathbf{\square}$

\title{
The Impact of Prior TURP on Radical Prostatectomy Surgical Margins: A Multicenter Analysis
}

C) S. Karger AG, Basel PROOF Copy
for personal
use only ANY DISTRIBUTION OF THIS ARTICLE WITHOUT WRITTEN CONSENT FROM S. KARGER AG, BASEL IS A VIOLATION OF THE COPYRIGHT.

\section{Mauro Gacci ${ }^{a} \quad$ Alchiede Simonato $^{c}$ Michele Lanciotti ${ }^{a} \quad$ Marco Ennas $^{c}$ Virginia Varca ${ }^{c}$ Massimo Maffezzini $^{d}$ Ciro $_{\text {Imbimbog }}{ }^{\text {Paolo }}$ Gontero $^{\text {h }}$ Riccardo Schiavina ${ }^{i}$} Marco Carini ${ }^{a}$ Giuseppe Martorana ${ }^{\mathrm{i}}$ Giulio Nicita ${ }^{\mathrm{b}}$ Vincenzo Mirone ${ }^{\mathrm{g}}$ Giorgio Carmignani $^{\mathrm{a}}$ and members of the MIRROR Study, LUNA Foundation

a Urologic Clinic 1 and b Urologic Clinic 2, University of Florence, Florence; ' 'L. Giuliani' Department of Urology,

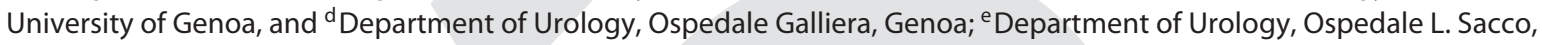
and ${ }^{f}$ IEO, Milan; ${ }^{9}$ Department of Urology, University of Naples Federico II, Naples; ${ }^{\text {h}}$ Urologic Clinic, University of Turin, Ospedale Molinette, Turin; 'Department of Urology, Alma Mater University Bologna, Bologna, Italy

\section{Key Words}

Prostate cancer $\cdot$ Radical prostatectomy $\cdot$ Positive surgical margins - Transurethral prostate resection

\begin{abstract}
Objective: To analyze positive surgical margins (PSM) after radical prostatectomy (RP) in the overall population and in patients previously treated with transurethral resection of the prostate (TURP). Materials and Methods: 2,408 patients treated with RP for clinically localized prostate cancer (PCa) were consecutively enrolled in 135 departments. We correlated PSM rates and all preoperative, surgical and pathological features. We stratified the site of PSM as unique or multifocal. Moreover, we analyzed differences between 75 patients who had undergone previous TURP and the remaining 2,333 patients. Results: In the entire study population, we identified 702 patients with PSM (29\%). Using univariate analysis, we reported a significant correlation between overall PSM and prostate-specific antigen (PSA), stage $\mathrm{CT}$, biopsy Gleason score, number of biopsy cores,
\end{abstract}

\section{KARGER}

(C) 2013 S. Karger AG, Basel

0042-1138/13/0000-0000\$38.00/0 number of positive cores, percentage of positive cores and nerve-sparing approach. PSM proved to be strongly dependent on $\mathrm{pT}$ in particular in patients with pT2 PCa. When we compared the data from 75 patients previously treated with TURP and those from 2,333 without previous prostatic surgery, a statistically significant difference in margin localization was found. Moreover, we analyzed the 75 patients mentioned above, stratified in incidental PCa diagnosed at TURP or PCa detected with prostate biopsy for PSA rising during the post-TURP follow-up: no statistical differences were found between the 2 groups regarding margin status, even if PSM were more frequent in incidental PCa with no significance deriving from the stratification for PSM location at the apex or base. Conclusion: Men treated with TURP before RP presented an overall incidence of PSM similar to those without previous TURP, but with a higher risk of PSM at the bladder neck and a lower risk of PSM at the prostatic apex.

Copyright $\odot 2013$ S. Karger AG, Basel

Dr. Mauro Gacci

Department of Urology, University of Florence, Careggi Hospital

Viale Pieraccini 19

IT-50139 Florence (Italy)

E-Mail maurogacci@yahoo.it 


\section{Introduction}

The overall incidence of prostate cancer $(\mathrm{PCa}) \mathrm{ac}$ counted for 186,000 new cases in the USA and 370,000 in Europe [1-3]; incidental PCa is found in $3-16 \%$ of specimens obtained from transurethral resection of the prostate (TURP) [4].

Radical prostatectomy (RP) represents the gold standard for clinically localized PCa [5], allowing to achieve 3 main outcomes ('trifecta'): cancer eradication, urinary continence restoration and erectile function recovery [6]. PCa can be diagnosed immediately after TURP or by a subsequent prostate-specific antigen (PSA) rise during the postoperative follow-up. Urologists can choose RP even after previous TURP, despite the subsequent alteration of the tissue layers due to fibrous scarring [7] and the increased risk of peri- and postoperative morbidity [8].

The safety and efficacy of RP after TURP are still debated. Only few studies have been made about it, assessing that TURP may cause a possible risk of positive surgical margins (PSM) $[6,9]$, especially in advanced stages [10]. To date, several studies conducted on large RP series did not systematically analyze post-TURP outcomes [11], even if a substantial percentage of men are found to have PCa after TURP [12].

In 2006 the Leading Urological No Profit Advanced Research (LUNA) Foundation planned the Multicenter Italian Report on Radical Prostatectomy Outcomes and Research (MIRROR) Study in order to evaluate the oncological, surgical and functional characteristics of PCa patients and RP outcomes.

The purpose of our study is to analyze PSM after RP in the overall population and in patients previously treated with TURP. Furthermore, we evaluated PSM after RP in men with incidental PCa (class pT1a-b) versus those with PCa discovered with a biopsy performed during postTURP follow-up for rising PSA.

\section{Materials and Methods}

\section{Patient Selection and Clinical Data}

In 2006 the LUNA Foundation planned the MIRROR Study. 135 urology departments all over Italy were contacted, to evaluate oncological and surgical characteristics of our study population of 2,408 patients treated for PCa. All patients were consecutively enrolled in each center and all data were prospectively recorded and stored by a web data manager (Clicon AG). For all patients the variables described below were recorded.

Preoperative Items. Age, body mass index, previous bladder outlet obstruction caused by clinically benign prostatic hyperplasia (acute urinary retention with catheterization and TURP) and pre- operative tumor characteristics (PSA value at the time of RP, clinical stage, and biopsy Gleason score, GS) were assessed.

Surgery. Nerve-sparing (NS) procedures (radical, monolateral, bilateral) were examined, while no data were available about the specific intra-, inter- or extrafascial technique. Bladder neck sparing was also investigated (yes or no).

Pathology. For pT, definitive GS, positive lymph nodes, and number and site of PSM, the pathological features were evaluated on whole formalin-fixed and paraffin-embedded surgical specimens that had been weighed and serially sectioned perpendicularly. The entire prostate was examined with 3-mm interval transections. pT was recorded according to the fifth UICC TNM classification. Tumor grade was assessed using the Gleason system. PSM were identified when tumor cells were seen in contact with the inked specimen surface. The presence, number and site of PSM were registered for each specimen. The locations of PSM were classified in: apex, base or bladder neck, lateral right, lateral left, posterior. Multifocal PSM was defined as the presence of neoplastic cells in $\geq 2$ sites among those reported above.

Population. 75 (3.1\%) patients treated with TURP before RP were stratified into 2 groups: group 1 included 32 (42.6\%) men with incidental PCa (pT1a-b) while group 2 included $43(57.4 \%)$ men who had undergone prostate biopsy during the follow-up after TURP, for rising PSA. Both groups were analogous for preoperative clinical and urological parameters. Prostate weight for all patients was assessed by ultrasound before TURP.

\section{Statistical Analyses}

A flow chart of the study population and statistical methods is reported in figure 1 . We assessed the overall incidence, number and site of PSM in the whole population of 2,408 patients. Within these groups we tested parameters for normality and correlated PSM rates and all preoperative variables, surgery and pathological features using a Spearman correlation coefficient. Significant data were included in a logistic regression analysis. Specific subanalyses regarding the NS procedure were performed with an unpaired sample $t$ test. Finally we stratified the site of PSM as unique or multifocal.

Out of the entire group of patients, 75 (3.1\%) had undergone a previous TURP and finally underwent RP in only 9 tertiary referral centers for treatment of PCa. Thus, we analyzed differences between the preoperative, surgical and oncological variables of these 75 (3.1\%) patients (with TURP) and the 2,333 (76.9\%) remaining patients (without TURP) with an unpaired sample $t$ test.

Moreover, we examined the 75 patients analyzing the differences between the $32(42.6 \%)$ patients with incidental PCa diagnosis at TURP (group 1) and the $43(57.4 \%$ ) patients who underwent prostate biopsy for PSA rise during the post-TURP follow-up (group 2) regarding pathological features (prostate weight, pT, GS, site and number of PSM), and early postoperative clinical outcomes were assessed by an unpaired sample $t$ test.

\section{Results}

The characteristics of our 2,408 patients are listed in table 1 . In the entire study population, we identified 702 patients with PSM (29\%). 464 of them (66\%) presented solitary PSM, while 238 (34\%) had multifocal PSM. In 


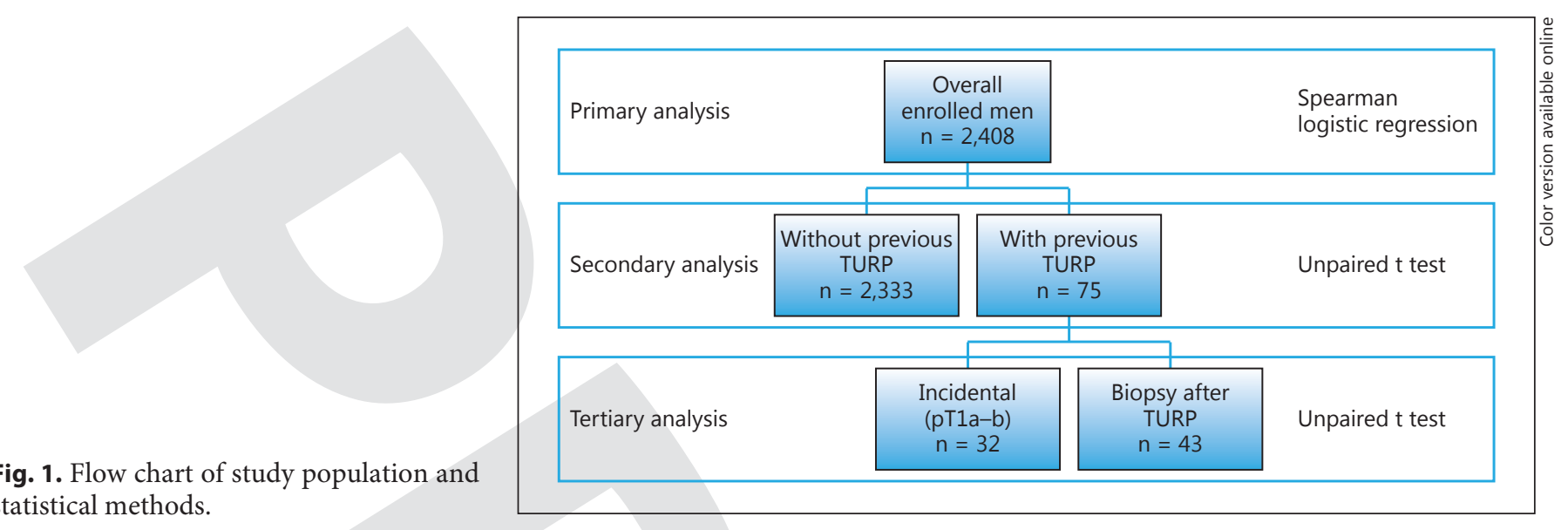

Fig. 1. Flow chart of study population and statistical methods.

$0.4074)$ and $\mathrm{pT}(\mathrm{p}=0.1773)$. In patients who had under-

particular, 167 patients presented PSM in 2 sites, 46 in 3 sites, 19 in 4 sites and 6 in 5 different sites.

Using univariate analysis with a Spearman correlation coefficient, we reported a correlation between overall PSM and PSA (coeff. -0.148; $\mathrm{p}=0.000$ ), cT (coeff. -0.128 ; $\mathrm{p}=0.000$ ), biopsy GS (coeff. $-0.153 ; \mathrm{p}=0.000$ ), number of biopsy cores (coeff. 0.066; $\mathrm{p}=0.001$ ), number of positive cores (coeff. $-0.134 ; \mathrm{p}=0.000$ ), percentage of positive cores (coeff. $-0.170 ; \mathrm{p}=0.000$ ) and NS approach (coeff. $0.254 ; \mathrm{p}=0.038$ ). PSM proved to be strongly dependent on $\mathrm{pT}$ (Spearman correlation coefficient $\mathrm{r}=-0.3422$; $\mathrm{p}<$ 0.0001): in particular, the rate of PSM in patients with pT2 PCa was $16.51 \%(253 / 1,532)$, compared to $51.26 \%$ $(449 / 876)$ in patients with advanced PCa ( $\geq$ pT3). These variables were included in the logistic regression models, and the results are reported in table 2.

We reported a different localization of single versus multifocal PSM according to margin location (table 3). In particular, the apex was frequently involved as a single PSM, while posterolateral locations (right or left) were involved more frequently in multifocal margins.

Stratifying the data of the overall population according to NS status, we performed fewer NS approaches in men with TURP compared to those without TURP (32.0 vs. $49.3 \%, p=0.0015)$, based on preoperative erectile activity. PSM were found in 407/1,234 (32.98\%) patients treated with a non-NS approach, in 75/318 (23.58\%) patients treated with monolateral NS surgery and 220/ 856 (25.70\%) patients treated with bilateral NS RP.

Secondary analyses comparing data from 75 patients (3.1\%) previously treated with TURP who finally underwent RP, and those from 2,333 without previous prostatic surgery demonstrated no difference according to preoperative PSA $(\mathrm{p}=0.2245)$, pathological GS $(\mathrm{p}=$

Impact of Prior TURP on Radical

Prostatectomy Surgical Margins gone a previous TURP, we reported a lower rate of NS approach compared to those without previous TURP $\left(\chi^{2}\right.$ $\mathrm{p}=0.0015$, $\mathrm{t}$ test $\mathrm{p}=0.038)$. After TURP, monolateral NS surgery was performed in $1.4 \%$ (vs. $13.6 \%$ without TURP), a bilateral one in $30.6 \%$ (vs. $35.7 \%$ ) and non-NS RP in $68 \%$ (vs. $50.7 \%$ ).

We did not identify a significant difference in the overall incidence of PSM between men with or without previous TURP (23 and $32 \%$, respectively, $\mathrm{p}=0.101$ ). On the other hand, we reported a significant difference in the sites of PSM: men with TURP presented a relatively higher rate of PSM compared to those without TURP at the bladder neck (5 vs. $2 \%, \mathrm{p}=0.049$ ) and a significantly lower rate of PSM at the apex ( 5 vs. $14 \%, \mathrm{p}=0.036$ ).

Moreover, in the tertiary analysis we evaluated 75 (3.1\%) patients with previous TURP, stratified in the 2 groups mentioned above: 32 patients (42.6\%) with a pT1a-b disease, and 43 patients (57.4\%) who underwent residual prostate biopsy long after TURP (groups 1 and 2, respectively). We evaluated several pathological items such as PSM, gland weight and GS. Above all, we found no statistical differences between the 2 groups regarding margin status ( $p=0.337)$, even if PSM were more frequent in incidental PCa (28.1\%) rather than in those who underwent a biopsy long after TURP (18.6\%), with no significance deriving from the stratification for PSM location at the apex or base.

Patients with incidental PCa at TURP presented a significantly lower prostate weight compared to those with biopsies performed several years after TURP (median weight: 27 vs. $40 \mathrm{~g}, \mathrm{p}=0.003$; fig. $2 \mathrm{a}$ ) and their mean GS was significantly lower ( $p=0.049$; fig. $2 b$ ) at the definitive pathological examination.

Urol Int

DOI: $10.1159 / 000346748$ 
Table 1. Descriptive statistics of the overall population $(2,408 \mathrm{pa}-$ tients)

\begin{tabular}{|c|c|c|}
\hline \multicolumn{3}{|l|}{ Clinical data } \\
\hline Age, years & $\begin{array}{l}\text { mean } \pm S D \\
\text { min. }-\max \end{array}$ & $\begin{array}{r}65.3 \pm 6.5 \\
31-85\end{array}$ \\
\hline \multirow[t]{2}{*}{ Age groups } & $<65$ years & 974 \\
\hline & $\geq 65$ years & 1,434 \\
\hline \multirow{2}{*}{ Body mass index } & mean $\pm S D$ & $26.3 \pm 3.0$ \\
\hline & $\min .-\max$ & $18.6-41.0$ \\
\hline \multicolumn{3}{|l|}{ Urological data } \\
\hline \multirow{2}{*}{$\begin{array}{l}\text { AUR with previous } \\
\text { catheterization }\end{array}$} & yes & 119 \\
\hline & no & 2,289 \\
\hline \multirow[t]{2}{*}{ Previous TURP } & yes & 75 \\
\hline & no & 2,333 \\
\hline \multicolumn{3}{|c|}{ Preoperative PCa characteristics } \\
\hline \multirow[t]{6}{*}{ PSA groups } & $0-2.4 \mathrm{ng} / \mathrm{ml}$ & 64 \\
\hline & $2.5-3.9 \mathrm{ng} / \mathrm{ml}$ & 190 \\
\hline & $4-10 \mathrm{ng} / \mathrm{ml}$ & 1,561 \\
\hline & $10.1-19.9 \mathrm{ng} / \mathrm{ml}$ & 433 \\
\hline & $>20 \mathrm{ng} / \mathrm{ml}$ & 160 \\
\hline & mean $\mathrm{PSA} \pm \mathrm{SD}$ & $9.7 \pm 12.9$ \\
\hline \multirow[t]{4}{*}{ Clinical stage (cT) } & T0-T1c & 1,131 \\
\hline & $\mathrm{T} 2$ & 957 \\
\hline & T3 & 95 \\
\hline & missed & 225 \\
\hline \multirow[t]{4}{*}{ Biopsy GS } & $<6$ & 247 \\
\hline & 6 & 1,285 \\
\hline & 7 & 651 \\
\hline & $8-10$ & 225 \\
\hline \multicolumn{3}{|l|}{ Operative features } \\
\hline \multirow[t]{3}{*}{ Nerve sparing } & non-NS & 1,234 \\
\hline & monolateral NS & 318 \\
\hline & bilateral NS & 856 \\
\hline \multirow[t]{2}{*}{ Bladder neck sparing } & yes & 1,938 \\
\hline & no & 470 \\
\hline \multicolumn{3}{|l|}{ Pathological outcomes } \\
\hline \multirow[t]{4}{*}{ Pathological stage } & $\mathrm{pT} 0$ & 11 \\
\hline & pT2 2 & 1,521 \\
\hline & pT3 & 856 \\
\hline & pT4 & 20 \\
\hline \multirow[t]{4}{*}{ Definitive GS } & $<6$ & 162 \\
\hline & 6 & 854 \\
\hline & 7 & 1,097 \\
\hline & $8-10$ & 295 \\
\hline \multirow[t]{3}{*}{ Lymph nodes } & $\mathrm{Nx}$ & 601 \\
\hline & No & 1,653 \\
\hline & $\mathrm{N} 1-2$ & 154 \\
\hline \multirow[t]{2}{*}{ Positive margins } & negative & 1,706 \\
\hline & positive & 702 \\
\hline
\end{tabular}

$\mathrm{AUR}=$ Acute urinary retention.

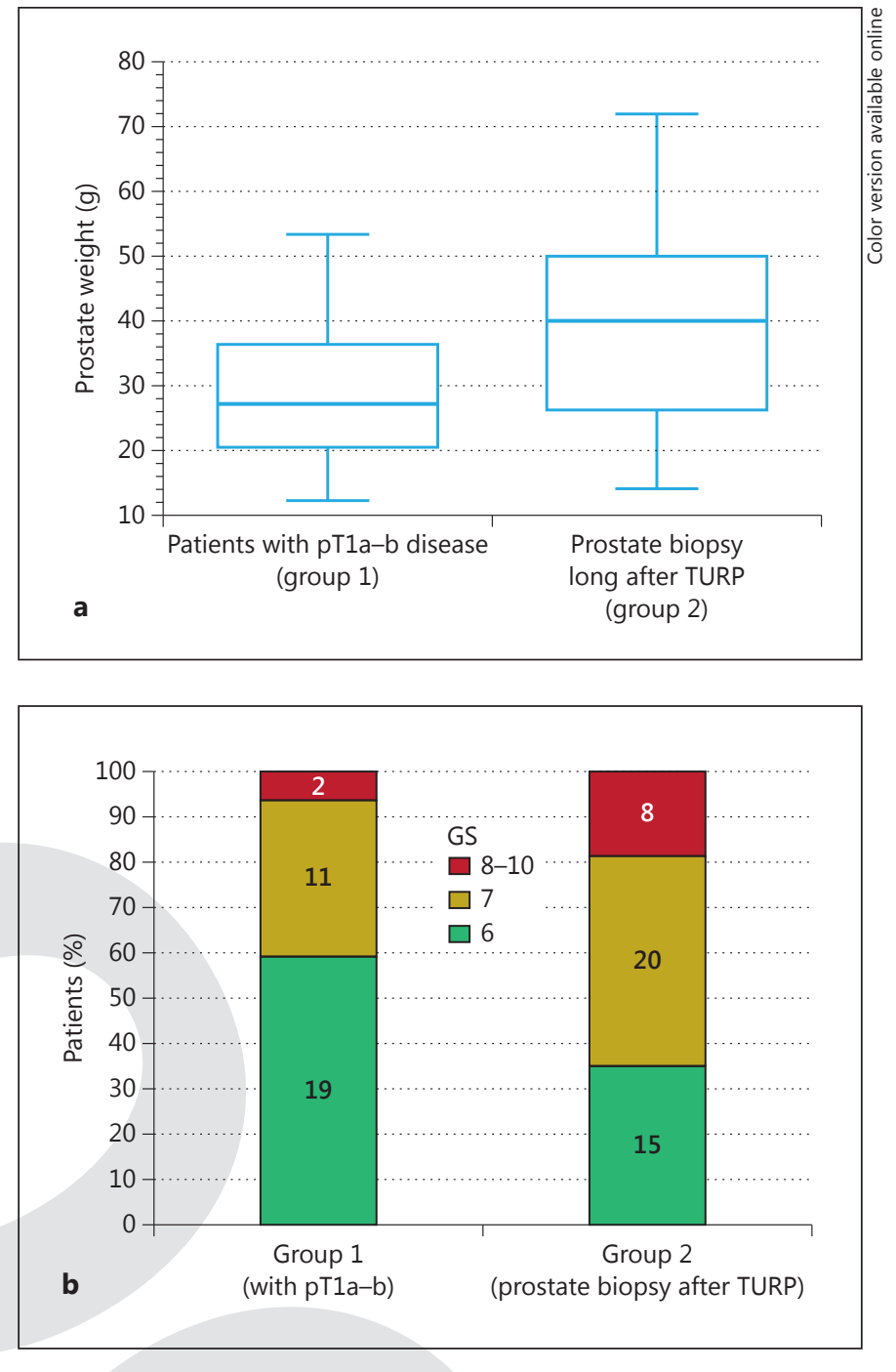

Fig. 2. Prostate weight of patients with incidental PCa at TURP compared to those with biopsies performed several years after TURP (a) and their mean GS (b) at the definitive pathological examination.

\section{Discussion}

PSM are mostly found in patients with higher stages, GS and PSA levels and occur in 11-38\% of the current series of RP [13]. It is commonly accepted that the most affected sites are the prostatic apex and posterolateral location [14]. In the MIRROR Study, PSM were strictly related to more advanced $\mathrm{pT}(\mathrm{p}<0.0001)$ as reported in the literature $[6,15]$. The global PSM rate in the MIRROR Study was 702/2,408 (29.1\%), more precisely 397 presented $(16.5 \%)$ pT2 and $1,234(51.2 \%) \geq$ pT3 stages. Overall
4

Urol Int

DOI: $10.1159 / 000346748$
Gacci et al. 
Table 2. Determinant of PSM in the overall population (2,408 patients)

\begin{tabular}{|c|c|c|c|c|c|}
\hline & \multicolumn{3}{|c|}{ Coefficients } & \multirow[t]{3}{*}{$\mathrm{t}$} & \multirow[t]{3}{*}{ Sig. } \\
\hline & \multicolumn{2}{|c|}{ non-standardized } & \multirow{2}{*}{$\begin{array}{l}\text { standardized } \\
\beta\end{array}$} & & \\
\hline & B & SE & & & \\
\hline Constant & 0.957 & 0.082 & & 11.660 & 0.000 \\
\hline Preoperative PSA & -5.425 & 0.000 & -0.004 & -0.171 & 0.864 \\
\hline Clinical stage $(\mathrm{cT})$ & -0.041 & 0.010 & -0.113 & -3.966 & $0.000^{*}$ \\
\hline Biopsy GS & -0.034 & 0.010 & -0.078 & -3.422 & $0.001^{*}$ \\
\hline Number of biopsy cores & 0.010 & 0.003 & 0.971 & 3.968 & $0.000^{*}$ \\
\hline Number of positive biopsy cores & -0.010 & 0.003 & -0.964 & -3.935 & $0.000^{*}$ \\
\hline Percent of positive biopsy cores & 0.023 & 0.021 & 0.028 & 1.097 & 0.273 \\
\hline NS & 0.026 & 0.011 & 0.051 & 2.259 & $0.024^{*}$ \\
\hline
\end{tabular}

Multivariate (logistic regression) analysis based on significant outcome at univariate analysis (Spearman correlation coefficient) are reported. Both the 'non-standardized coefficient' and the corrected 'standardized coefficient' are reported. ${ }^{*} \mathrm{p}<0.05$.

Table 3. PSM in the overall population (2,408 patients)

\begin{tabular}{llllllr}
\hline & Overall & Apex & Base & Posterior & Lateral right & Lateral left \\
\hline All & $702(29 \%)$ & 325 & 51 & 132 & 281 & 253 \\
Unique & $464(66 \%)$ & 177 & 23 & 60 & 113 & 91 \\
Multifocal & $238(34 \%)$ & 148 & 28 & 72 & 168 & 162 \\
\hline
\end{tabular}

Data are stratified according to number of PSM (unique or multifocal) and site of PSM (apex, base, posterior, lateral right, lateral left).

PSM could be reduced with a more accurate case selection, practicing RP in small tumors (lower stages) and adopting an NS technique after considering age, preoperative erections, PSA, clinical stage and GS [15-18].

Some authors assert that the incidence of PSM is similar among open, laparoscopic and robotic RP (LRP and RALRP) techniques [13], while other authors suggest that for mini-invasive techniques it may be influenced mainly by the operator learning curve [15]. Large series show that PSM range between 6 and $31 \%$ in the retropubic RP [16$18], 12.6-27 \%$ in the laparoscopic approach $[9,10,19]$ and $13.5-15.2 \%$ in the robotic technique $[15,20]$. Also the employment of an NS or non-NS technique does not result in significant differences in PSM rates: 5.9-24\% PSM in NS groups, $5.8-31 \%$ in non-NS groups [16-18]. However, this data can be strongly biased by patient selection for mini-invasive procedures and by the learning curve for LRP and RALRP.

Impact of Prior TURP on Radical

Prostatectomy Surgical Margins
RP after TURP can be currently performed by skilled urologists, especially in young men with suspected clinically localized PCa [6-10] with radical intent. In patients without previous TURP, with favorable clinical predictive factors, surgeons generally perform RP with NS techniques, and for this reason the margins of resection are very close to the prostate. In contrast, in men with previous TURP, even those with favorable clinical features, surgeons would perform RP with a wider margin of resection; this could explain the overall lower rate of PSM that we have found in our population after TURP, even if this data was not statistically significant. Concerning this, we report a lower rate of NS techniques performed in men with TURP compared to those without TURP (32.0 vs. $49.3 \%, p=0.0015)$, in accordance with data reported in the current literature [20].

Thus, a previous TURP did not seem to influence the overall PSM rate, in accordance with the literature. Also in large series of LRP and RALRP, no statistical signifi-

Urol Int

DOI: $10.1159 / 000346748$ 
cance was found when comparing groups subjected to previous TURP or not, concluding that performing RP after TURP does not increase the perioperative complication rate $[6,10,20]$. To our knowledge the only discordant results have been obtained by Jaffe et al. [9], who suggested that a history of benign prostatic hyperplasia could delay the diagnosis of a synchronous cancer explaining the less favorable rate of PSM in their TURP group.

TURP was related to a higher risk of PSM at the bladder neck and a lower rate at the apex, as compared to men without TURP ( 5 vs. $2 \%$ and 5 vs. $14 \%$, respectively). These outcomes are in part in accordance with those previously reported by Shelfo et al. [21].

After the removal of large adenomas protruding into the bladder, the bladder neck can be definitively widened and thinned. Therefore, during the subsequent RP, surgeons can shift the margins of the bladder neck incision into the prostatic base, with the improved risk of PSM. In contrast, the huge fibrotic processes at the prostatic apex after TURP can induce the surgeon to put forward the point of apical resection with the consequent low risk of PSM.

In our tertiary subanalysis, we demonstrated that across 75 men treated with previous TURP, those with incidental pT1a-b presented prostates with lower volume and lower GS as compared with those who had had a biopsy long after TURP (fig. 2). The reduced prostatic volume immediately after TURP can be due to the lack of time for regrowth of prostatic tissue after TURP. In addition, younger men were selected for the treatment of benign prostatic hyperplasia, presenting prostate volumes of $30-80 \mathrm{ml}$ according to the current guidelines $[22,23]$, and as reported by Secin et al. [24] a prostate volume of $\leq 30 \mathrm{~g}$ was associated with a greater risk of PSM after LRP.

It was an expected data to find higher GS PCa after TURP that were candidates for RP, as it is well documented in the literature that a high GS is associated with greater tumor volume and positive margins $[25,26]$, and probably because of the incidental low risk (low GS) elderly patients were selected for active surveillance according to AUA guidelines [27]. As it has already been suggested in the literature, Donovan et al. [28] demonstrated that GS had a significant role in their predictive model constructed on clinical data and features extracted from TURP tissue specimens, given the high percentage of events (79\%) with GS $\geq 7$. Furthermore there are no reports in the literature concerning these topics and further evaluations and studies are needed to clarify this issue.
A possible limitation of our survey is the relatively higher incidence of PSM, probably due to the multicentric typology of design of the study, including an elevated number of surgeons from primary to tertiary surgical centers. Indeed inclusion criteria were not set to include divisions of urology in order to survey the national situation concerning RP. Our mean follow-up time was still short to set out relevant data concerning biochemical and disease-free outcomes. Furthermore, the prostate specimens were not analyzed by the same pathologist, and even if there are some definitions of PSM that are adopted as guidelines, PSM does not, in any case, merely depend on the biology of the cancer or on surgical factors, and it is also determined by adequate specimen handling and accurate interpretation [13]. Thus, it is difficult to compare our heterogeneous data with the literature which is produced, in general, by individual divisions of urology well focused on this subject.

\section{Conclusions}

The apex is the most frequent site of single PSM, while lateral margins are frequently involved in multifocal PSM. Clinical stage, biopsy GS and the number of both overall and positive biopsy cores are the main risk factors for PSM. RP after a previous TURP seems to be a safe treatment for PCa, with an overall incidence of PSM similar to those reported in men without previous TURP. Nevertheless, TURP is associated with a higher risk of PSM at the bladder neck and a lower risk at the prostatic apex. Men treated with RP in incidental PCa (pT1a-b) have a reduced prostate volume with a lower GS as compared with those who underwent biopsy long after TURP.

\section{Acknowledgment}

The authors would like to thank Lisa Marie Terzariol for the careful linguistic revision of the manuscript.

\section{Disclosure Statement}

The authors have no conflicts of interest to disclose. 


\section{References}

1 Jemal A, Siegel R, Ward E, et al: Cancer statistics, 2008. CA Cancer J Clin 2008;58:71-96.

2 Ferlay J, Shin HR, Bray F, et al: GLOBOCAN 2008 v1.2, Cancer Incidence and Mortality Worldwide: IARC Cancer Base No 10. 2010. http://globocan.iarc.fr (accessed May 18, 2012)

3 Maffezzini M: The magnitude of the problem and the burden of physicians and patients decisions. Surg Oncol 2009;18:183-184.

4 Kanno H, Umemoto S, Izumi K, et al: Prostate cancer development after transurethral resection of the prostate: histopathological studies of radical prostatectomy specimens. Jpn J Urol 2006;97:649-659.

5 Schaeffer EM, Loeb S, Walsh PC: The case for open radical prostatectomy. Urol Clin North Am 2010;37:49-55.

6 Bianco FJ Jr, Scardino PT, Eastham JA: Radical prostatectomy: long-term cancer control and recovery of sexual and urinary function ('trifecta'). Urology 2005;66:83-94.

7 Palisaar JR, Wenske S, Sommerer F, et al: Open radical retropubic prostatectomy gives favourable surgical and functional outcomes after transurethral resection of the prostate. BJU Int 2009;104:611-615.

8 Teber D, Cresswell J, Ates M, et al: Laparoscopic radical prostatectomy in clinical Tla and T1b prostate cancer: oncologic and functional outcomes - a matched-pair analysis. Urology 2009;73:577-581.

9 Jaffe J, Stakhovsky O, Cathelineau X, et al: Surgical outcomes for men undergoing laparoscopic radical prostatectomy after transurethral resection of the prostate. J Urol 2007; 178:483-487.

10 Menard J, de la Taille A, Hoznek A, et al: Laparoscopic radical prostatectomy after transurethral resection of the prostate: surgical and functional outcomes. Urology 2008;72:593597.
11 Bott SR, Freeman AA, Stenning S, et al: Radical prostatectomy: pathology findings in 1,001 cases compared with other major series and over time. BJU Int 2005;95:34-39.

12 Bright EA, Manuel C, Goddard JC, et al: Incidence and factors predicting the detection of prostate cancer after transurethral resection of the prostate for clinically benign disease. Urol Int 2009;83:171-174.

13 Yossepowitch O, Bjartell A, Eastham JA, et al: Positive surgical margins in radical prostatectomy: outlining the problem and its longterm consequences. Eur Urol 2009;55:87-99.

14 Blute ML, Bostwick DG, Bergstralh EJ, et al: Anatomic site-specific positive margins in organ-confined prostate cancer and its impact on outcome after radical prostatectomy. Urology 1997;50:733-739.

15 Gettman MT, Blute ML: Radical prostatectomy: does surgical technique influence margin control? Urol Oncol 2010;28:219-225.

16 Hernandez DJ, Epstein J, Trock BJ, et al: Radical retropubic prostatectomy. How often do experienced surgeons have positive surgical margins when there is extraprostatic extension in the region of the neurovascular bundle? J Urol 2005;173:446-449.

17 Sofer M, Hamilton-Nelson KL, Schlesselman $\mathrm{JJ}$, et al: Risk of positive margins and biochemical recurrence in relation to nerve-sparing radical prostatectomy. J Clin Oncol 2002; 20:1853-1858.

18 Palisaar RJ, Noldus J, Graefen M, et al: Influence of nerve-sparing (NS) procedure during radical prostatectomy $(\mathrm{RP})$ on margin status and biochemical failure. Eur Urol 2005;47: 176-184.

19 Frota R, Stein RJ, Turna B, et al: Are prostate needle biopsies predictive of the laterality of significant cancer and positive surgical margins? BJU Int 2009;104:1599-1603.

20 Do M, Haefer T, Liatsikos E, et al: Endoscopic extraperitoneal radical prostatectomy after previous transurethral resection of prostate: oncologic and functional outcomes of 100 cases. Urology 2010;75:1348-1352.
21 Shelfo SW, Obek C, Soloway MS: Update on bladder neck preservation during radical retropubic prostatectomy: impact on pathologic outcome, anastomotic strictures, and continence. Urology 1998;51:73-78.

22 McVary KT, Roehrborn CG, Avins AL, Barry MJ, Bruskewitz RC, Donnell RF, Foster HE, Gonzalez CM, Kaplan SA, Penson DF, Ulchaker JC, Wei JT: AUA Clinical Guidelines: Management of $\mathrm{BPH}$, rev ed.

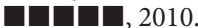

23 Oelke M, Alivizatos G, Emberton M, et al Pocket Guideline: Guidelines on Benign Prostatic Hyperplasia. Association of Urology, 2011.

24 Secin FP, Serio A, Bianco FJ Jr, et al: Preoperative and intraoperative risk factors for side-specific positive surgical margins in laparoscopic radical prostatectomy for prostate cancer. Eur Urol 2007;51:764-771.

25 Stamey TA, Villers AA, McNeal JE, Link PC, Freiha FS: Positive surgical margins at radical prostatectomy: importance of apical dissection. J Urol 1990;143:1166-1173.

26 Jones EC: Resection margin status in radical retropubic prostatectomy specimens: relationship to type of operation, tumor size, tumor grade and local tumor extension. J Urol 1990;144:89-93.

27 Thompson I, Thrasher JB, Aus G, Burnett AL, Canby-Hagino ED, Cookson MS, D'Amico AV, Dmochowski RR, Eton DT, Forman JD, Goldenberg SL, Hernandez J, Higano CF Kraus SR, Moul JW, Tangen CM: AUA Clir ical Guidelines: Prostate Cancer, rev ed.

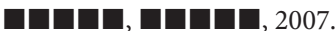

28 Donovan MJ, Khan FM, Bayer-Zubek V, Powell D, Costa J, Cordon-Cardo C: A systems-based modelling approach using transurethral resection of the prostate (TURP) specimens yielded incremental prognostic significance to Gleason when predicting longterm outcome in men with localized prostate cancer. BJU Int 2012;109:207-213.
Impact of Prior TURP on Radical

Prostatectomy Surgical Margins
Urol Int

DOI: $10.1159 / 000346748$ 